\title{
Espécies de Cerambycidae, Disteniidae e Vesperidae (Insecta, Coleoptera) registradas no Estado do Mato Grosso do Sul, Brasil
}

\author{
Miguel Angel Monné', Marcela Laura Monné1,2, Juan Pablo Botero', \\ Vanessa Souza Machado', Allan Carelli', Mario Cupello' \& Rodrigo Souza'
}

\begin{abstract}
1. Departamento de Entomologia, Museu Nacional, Universidade Federal do Rio de Janeiro, UFRJ. Quinta da Boa Vista, São Cristóvão, 20940-040, Rio de Janeiro, RJ, Brasil. (monne@uol.com.br)
\end{abstract}

Recebido 21 novembro 2016

Aceito 6 fevereiro 2017

DOI: 10.1590/1678-4766e2017119

ABSTRACT. Cerambycidae, Disteniidae and Vesperidae species (Insecta, Coleoptera) recorded from Mato Grosso do Sul State, Brazil. A survey of the Cerambycidae, Disteniidae and Vesperidae species recorded from Mato Grosso do Sul state, Brazil, is presented. The data were based on literature and in the collection of the Museu Nacional, Universidade Federal do Rio de Janeiro. Two hundred and twenty nine species are registered to Cerambycidae, distributed in five subfamilies. Two species of Disteniidae and two of Vesperidae, are registered.

KEYWORDS. Checklist, inventory, Neotropical, Biota-MS Program.

RESUMO. É apresentado um levantamento das espécies de Cerambycidae, Disteniidae e Vesperidae que ocorrem no estado do Mato Grosso do Sul, Brasil. Os dados foram baseados na literatura e no acervo da coleção do Museu Nacional, Universidade Federal do Rio de Janeiro. São registradas 229 espécies de Cerambycidae, distribuídas em cinco subfamílias. São registradas duas espécies de Disteniidae e duas de Vesperidae.

PALAVRAS-CHAVE. Lista, Inventário, Neotropical, Programa Biota-MS.

O estudo da sistemática de Cerambycidae e famílias afins é fundamental para profissionais das áreas florestal, frutícola e conservacionista, pois constituem uma família cujas larvas são xilófagas e broqueiam troncos e galhos, podendo causar danos econômicos. Além disso, são fundamentais no processo de decomposição da madeira morta, incorporando ao solo as partes vegetais e permitindo a renovação das florestas através da abertura de espaços e consequente germinação das sementes. Outro ponto importante é que existe uma complexidade de formas e padrões evolutivos que começam a ser explorados e estes padrões estão representados nas relações filogenéticas (NAPP, 1994; LiNGAFELTER, 1998; MonNÉ \& NAPP, 2005) e biogeográficas (PhILIPS \& IVIE, 1998; MonNÉ, 2005).

Este trabalho tem como objetivo fornecer, pela primeira vez, uma lista das espécies de Cerambycidae, Disteniidae e Vesperidae registradas para o estado do Mato Grosso do Sul, Brasil, com base em dados da literatura e da coleção de Coleoptera do Museu Nacional, Universidade Federal do Rio de Janeiro.

\section{MATERIAL E MÉTODOS}

Foram estudados os exemplares da coleção de Coleoptera do Museu Nacional, Universidade Federal do
Rio de Janeiro com registro para o estado do Mato Grosso do Sul e a literatura pertinente foi examinada com intuito de complementar o estudo.

A lista é apresentada em ordem alfabética de família, subfamília, tribo, subtribo, gênero e espécie e as referências sob cada espécie restringem-se à descrição original e à dos autores que citaram a espécie para o estado do Mato Grosso do Sul. O item distribuição, em cada espécie, foi obtido de MonNÉ $(2005 ; 2006)$ e todos os trabalhos posteriores, até julho de 2012. O item material examinado foi incluído quando existia registro na coleção do Museu Nacional, Universidade Federal do Rio de Janeiro. Sigla utilizada no texto: MNRJ, Museu Nacional, Universidade Federal do Rio de Janeiro, Rio de Janeiro, Brasil.

\section{RESULTADOS}

São registradas 229 espécies de Cerambycidae, em cinco subfamílias. A subfamília Cerambycinae foi a que apresentou mais registros, 166 espécies, seguida de Lamiinae com 57 espécies; em Prioninae registramos apenas quatro espécies: Prionapterus travassosi Lane, 1938, Calocomus morosus White, 1850, Calocomus rugosipennis Lucas, 1857 e Pyrodes nitidus (Fabricius, 1787); em Lepturinae apenas Strangalia melanophthisis (Berg, 1889); e em Parandrinae 
somente Parandra (Parandra) glabra (DeGeer, 1774).

Em Disteniidae registramos Cometes hirticornis Lepeletier \& Audinet-Serville, 1828 e Distenia (Basisvallis) striaticollis Villiers, 1959, e em Vesperidae Anoploderma breueri Lameere, 1912 e Migdolus spitzi Lane, 1937.

\section{CERAMBYCIDAF}

CERAMBYCINAE

ACHRYSONINI

1. Achryson maculatum Burmeister, 1865. Distribuição: Brasil (Mato Grosso do Sul, Espírito Santo ao Rio Grande do Sul), Bolívia, Paraguai, Argentina, Uruguai. Mato Grosso do Sul: Brasilândia (Rio Taquarussu); Corumbá, Corumbá (Serra do Urucum); Três Lagoas (Fazenda Canaã, margem esquerda do Rio Sucuriú).

\section{CALLICHROMATINI}

2. Cnemidochroma phyllopoides (Schmidt, 1924). Distribuição: Brasil (Mato Grosso, Mato Grosso do Sul, Goiás). Mato Grosso do Sul: Corumbá.

3. Cnemidochroma phyllopus (Guérin-Méneville, 1844). Distribuição: Brasil (Bahia a Rio Grande do Sul, Mato Grosso do Sul), Paraguai, Argentina (Misiones, Santa Fé, Entre Ríos), Uruguai. Mato Grosso do Sul: Bataguassu (Rio Caraguatá - 400m). 4. Mionochroma equestre (Gounelle, 1911). Distribuição: Brasil (Mato Grosso do Sul, Goiás, Minas Gerais ao Rio de Janeiro), Paraguai, Argentina (Misiones). Mato Grosso do Sul: Brasilândia (Rio Taquarussu) (NAPP \& MarTins, 2009:29).

\section{CERAMBYCINI}

CERAMBYCINA

5. Hamaticherus bellator Audinet-Serville, 1834. Distribuição: Equador, Guiana Francesa, Guiana, Suriname, Brasil (Amazonas, Pará, Goiás, Maranhão, Bahia, Minas Gerais, Mato Grosso do Sul, São Paulo). Mato Grosso do Sul - Miranda (Salobra) 6. Plocaederus confusus Martins \& Monné, 2002. Distribuição: Brasil (Ceará, Mato Grosso, Goiás, Maranhão, Mato Grosso do Sul, Bahia ao Paraná), Bolívia.

Mato Grosso do Sul - Bataguassu (Rio Caraguatá - 400m); Brasilândia (Rio Taquarussu).

SPHALLOTRICHINA

7. Coleoxestia cinnamomea (Gounelle, 1909). Distribuição: Brasil (Goiás, Mato Grosso do Sul, São Paulo), Paraguai, Argentina (Corrientes, Entre Ríos). Mato Grosso do Sul: Maracaju; Porto Murtinho (MarTins \& MonNÉ, 2005:148).

8. Coleoxestia corvina (Germar, 1824). Distribuição: Brasil (Rondônia, Mato Grosso, Goiás, Maranhão, Pernambuco ao Rio Grande do Sul, Mato Grosso do Sul), Bolívia, Paraguai, Argentina (Jujuy, Santiago del Estero, Tucumán, Misiones, Chaco, Corrientes), Uruguai. Mato Grosso do Sul: Costa Rica (Fazenda Santo Antônio); Coxim; Miranda (Salobra); Porto Murtinho (MARTINs \& MonNÉ,2005:131)

9. Coleoxestia denticornis (Gahan, 1892). Distribuição: Brasil (Goiás, Mato Grosso do Sul, Paraíba, Pernambuco, Bahia, Espírito Santo ao Rio Grande do Sul), Bolívia, Paraguai. Mato Grosso do Sul: Porto Murtinho (MarTins \& MonNé, 2005:152).

10. Coleoxestia ebenina Melzer, 1935. Distribuição: Brasil (Mato Grosso, Mato Grosso do Sul, Goiás, Minas Gerais, São Paulo), Bolívia, Argentina (Misiones). Mato Grosso do Sul: Porto Murtinho (Fazenda Várzea Alegre) (localidade-tipo) (MARTINS \& MonNÉ, 2005:149).

11. Coleoxestia pubicornis (Gounelle, 1909). Distribuição: Brasil (Goiás, Mato Grosso do Sul, Pernambuco ao Rio Grande do Sul), Bolívia. Mato Grosso do Sul: Porto Murtinho (Martins \& MonNÉ, 2005:174).

12. Criodion torticolle Bates, 1870. Distribuição: Colômbia, Venezuela, Peru, Guiana Francesa, Brasil (Amazonas, Pará, Maranhão, Ceará, Mato Grosso do Sul, São Paulo), Bolívia, Paraguai, Argentina (Formosa, Santiago del Estero). Mato Grosso do Sul: Miranda (Salobra) (Martins \& Monné, 2005:203).

13. Poeciloxestia lanei Fragoso, 1978. Distribuição: Brasil (Mato Grosso do Sul), Bolívia. Mato Grosso do Sul: Ladário. Material examinado: BRASIL, Mato Grosso do Sul: Ladário, $\widehat{\partial}$ (holótipo), X.1969, A. Azevedo col. (MNRJ).
14. Poeciloxestia parallela Fragoso, 1978. Distribuição: Brasil (Pará, Mato Grosso do Sul, Goiás, Minas Gerais a São Paulo). Mato Grosso do Sul: Costa Rica (Martins \& MonNé, 2005:99). 15. Poeciloxestia suturalis (Perty, 1832). Distribuição: Brasil (Maranhão, Goiás, Mato Grosso, Mato Grosso do Sul, Minas Gerais, Rio de Janeiro ao Rio Grande do Sul), Bolívia, Paraguai, Argentina (Salta, Jujuy, Tucumán). Mato Grosso do Sul: Miranda (Salobra); Porto Murtinho (Martins \& MonNÉ, 2005:94).

\section{CLYTINI}

16. Cotyclytus curvatus (Germar, 1821). Distribuição: Brasil (Mato Grosso do Sul, Goiás, Bahia ao Rio Grande do Sul), Paraguai, Argentina (Misiones, Formosa, Chaco, Corrientes, Entre Ríos, Buenos Aires), Uruguai. Mato Grosso do Sul: Brasilândia (Rio Taquarussu) (Martins \& Galileo, 2011:138).

17. Megacyllene acuta (Germar, 1821). Distribuição: Brasil (Goiás, Mato Grosso, Mato Grosso do Sul, Bahia ao Rio Grande do Sul), Bolívia, Paraguai, Argentina (Salta, Tucumán, Catamarca, Chaco, Mendoza, Santa Fé, Misiones, Corrientes, Entre Ríos, Buenos Aires), Uruguai. Mato Grosso do Sul: Batayporã (Porto Primavera); Caarapó (Fazenda Pai Cué); Corumbá (Santa Branca); Iguatemi; Nova Andradina; Três Lagoas (Fazenda Floresta) (MarTins \& Galileo, 2011:28).

\section{COMPSOCERINI}

18. Aglaoschema dulce (Napp \& Martins, 1988). Distribuição: Brasil (Mato Grosso do Sul); Bolívia. Mato Grosso do Sul: Corumbá (Urucum) (localidade-tipo).

19. Aglaoschema ventrale (Germar, 1824). Distribuição: Brasil (Goiás, Mato Grosso do Sul, Bahia ao Rio Grande do Sul), Bolívia, Paraguai, Argentina (Misiones). Mato Grosso do Sul: Dourados (NAPP, 2007a:802)

20. Chlorethe brachyptera Zajciw, 1963. Distribuição - Brasil (Mato Grosso do Sul, Paraná, Santa Catarina), Bolívia. Mato Grosso do Sul: Bataguassu (Rio Caraguatá - 400m). Material examinado: BRASIL, Mato Grosso do Sul: Bataguassu (Rio Caraguatá 400m, 21 ${ }^{\circ} 48^{\prime} \mathrm{B} 52^{\circ} 27^{\prime} \mathrm{L}$ ), ơ (parátipo), II.1953, F. Plaumann col.; (parátipo), XII.1953, mesma localidade e coletor (MNRJ). 21. Chlorethe scabrosa Zajciw, 1963. Distribuição: Brasil (Mato Grosso do Sul, Espírito Santo, Paraná, Santa Catarina, Rio Grande do Sul), Argentina (Misiones). Mato Grosso do Sul: Bataguassu (Rio Caraguatá $-400 \mathrm{~m}$ ). Material examinado: BRASIL, Mato Grosso do Sul: Bataguassu (Rio Caraguatá - 400m, $21^{\circ} 48^{\prime} \mathrm{B}$ $52^{\circ} 27^{\prime} \mathrm{L}$ ), ôe 4 ( (parátipos) III.1953 F. Plaumann col. (MNRJ). 22. Cosmoplatidius simulans (Bates, 1870). Distribuição: Equador, Peru, Brasil (Amazonas, Rondônia, Mato Grosso do Sul), Bolívia. Mato Grosso do Sul: Corumbá (NAPP \& MarTins, 2006:472). 23. Orthostoma abdominale (Gyllenhal, 1817). Distribuição: Colômbia, Equador, Peru, Brasil (Amazonas, Pará, Rondônia, Mato Grosso, Mato Grosso do Sul, Goiás, Bahia ao Rio Grande do Sul), Bolívia, Paraguai, Argentina (Santiago del Estero, Misiones).Mato Grosso do Sul: Três Lagoas (Fazenda Beija-Flor).

\section{DIORINI}

24. Diorus biapiculatus White, 1853. Distribuição: Brasil (Goiás, Mato Grosso, Espírito Santo, São Paulo, Mato Grosso do Sul, Rio Grande do Sul), Paraguai. Mato Grosso do Sul: Miranda (Salobra).

EBURIINI

25. Beraba cheilaria (Martins, 1967). Distribuição: Brasil (Mato Grosso do Sul), Bolívia. Mato Grosso do Sul: Miranda (Salobra - Estrada de Ferro Noroeste do Brasil) (localidade-tipo). 26. Cupanoscelis heteroclita Gounelle, 1909. Distribuição: Brasil (Mato Grosso, Goiás, Mato Grosso do Sul, Maranhão, Piauí, Pernambuco, Minas Gerais, São Paulo), Bolívia, Paraguai, Argentina (Santiago del Estero, Córdoba). Mato Grosso do Sul: Três Lagoas (Fazenda Beija-Flor)

27. Eburella pumicosa Monné \& Martins, 1973. Distribuição: Brasil (Mato Grosso, Mato Grosso do Sul), Bolívia, Paraguai. Mato Grosso do Sul: Miranda (Salobra) (localidade-tipo); Porto Murtinho (Joaquim Murtinho); Selvíria (Fazenda da Unesp). 28. Eburodacrys assimilis Gounelle, 1909. Distribuição: Brasil (Piauí, Maranhão, Mato Grosso, Goiás, Mato Grosso do Sul, São Paulo), Bolívia. Mato Grosso do Sul: Três Lagoas (Fazenda Beija-flor), Fazenda Yamaguti, Córrego da Onça. 
29. Eburodacrys crassimana Gounelle, 1909. Distribuição: Suriname, Brasil (Pará, Maranhão, Mato Grosso, Goiás, Mato Grosso do Sul, Piauí a Santa Catarina), Bolívia, Paraguai, Argentina (Catamarca, Santiago del Estero, Mendoza, Misiones, Chaco). Mato Grosso do Sul: Coxim; Miranda (Salobra).

30. Eburodacrys cunusaia Martins, 1997. Distribuição: Brasil (Maranhão, Mato Grosso, Mato Grosso do Sul, Goiás, Paraíba, Bahia, Minas Gerais), Paraguai. Mato Grosso do Sul: Miranda (Salobra).

31. Eburodacrys dubitata White, 1853. Distribuição: Brasil (Maranhão, Goiás, Mato Grosso do Sul, Bahia ao Rio Grande do Sul), Bolívia, Paraguai, Argentina (Misiones, Buenos Aires), Uruguai. Mato Grosso do Sul: Miranda (Salobra); Três Lagoas (Fazenda Beija-Flor).

32. Eburodacrys elegantula Gounelle, 1909. Distribuição: Brasil (Mato Grosso, Goiás, Mato Grosso do Sul), Bolívia. Mato Grosso do Sul: Brasilândia (Rio Taquarussu); Porto Murtinho (Joaquim Murtinho).

33. Eburodacrys flexuosa Gounelle, 1909. Distribuição: Brasil (Mato Grosso, Mato Grosso do Sul, Goiás, Paraíba a São Paulo), Bolívia, Paraguai, Argentina (Salta, Chaco). Mato Grosso do Sul: Campo Grande; Miranda (Salobra).

34. Eburodacrys fortunata Lameere, 1884. Distribuição: Brasil (Mato Grosso, Mato Grosso do Sul, Goiás, Maranhão, Piauí a Santa Catarina), Paraguai. Mato Grosso do Sul: Miranda (Salobra). 35. Eburodacrys havanensis Chevrolat, 1862. Distribuição: Cuba, México a Nicarágua, Costa Rica, Panamá, Venezuela, Bolívia, Brasil (Mato Grosso, Mato Grosso do Sul, Goiás, Maranhão, Piauí a Santa Catarina), Bolívia, Paraguai. Mato Grosso do Sul: Miranda (Salobra).

36. Eburodacrys punctipennis White, 1853. Distribuição: Brasil (Mato Grosso, Mato Grosso do Sul, Goiás, Bahia ao Paraná), Paraguai. Mato Grosso do Sul: sem localidade específica para o estado.

37. Eburodacrys sexmaculata (Olivier, 1790). Distribuição: Venezuela, Equador, Suriname, Guiana, Guiana Francesa, Peru, Bolívia, Brasil (Amazonas ao Rio Grande do Sul, Mato Grosso do Sul), Bolívia. Mato Grosso do Sul: sem localidade específica para o estado.

38. Eburodacrys stahli Aurivillius, 1893. Distribuição: Bolívia, Brasil (Mato Grosso, Mato Grosso do Sul, Goiás, Maranhão, Minas Gerais ao Rio Grande do Sul), Bolívia, Argentina (Misiones), Paraguai, Uruguai. Mato Grosso do Sul: Miranda (Salobra). 39. Eburodacrys truncata Fuchs, 1956. Distribuição: Brasil (Maranhão, Mato Grosso, Mato Grosso do Sul, Goiás, Piauí ao São Paulo), Paraguai. Mato Grosso do Sul: Bataguassu (Rio Caraguatá) (localidade-tipo); Caarapó (Santa Luzia, ex-Juti); Miranda (Salobra).

40. Eburodacrys tuberosa Gounelle, 1909. Distribuição: Brasil (Goiás, Mato Grosso do Sul, Minas Gerais, São Paulo), Argentina. Mato Grosso do Sul: Miranda (Salobra).

41. Eburodacrys vittata (Blanchard, 1846). Distribuição: Brasil (Paraíba a Santa Catarina, Mato Grosso do Sul.), Bolívia, Paraguai, Argentina, Uruguai. Mato Grosso do Sul: Miranda (Salobra). 42. Erosida formosa (Blanchard, 1846). Distribuição: Brasil (Pará, Rondônia, Maranhão, Mato Grosso do Sul), Bolívia. Mato Grosso do Sul: Corumbá (Serra do Urucum).

43. Erosida lineola (Fabricius, 1781). Distribuição: Brasil (Rio Grande do Norte, Mato Grosso do Sul, Goiás, Minas Gerais a Santa Catarina), Paraguai, Uruguai. Mato Grosso do Sul: Brasilândia (Rio Taquarussu); Miranda (Salobra).

44. Pronuba lenkoi Monné \& Martins, 1974. Distribuição: Brasil (Mato Grosso do Sul), Bolívia. Mato Grosso do Sul: Corumbá (Serra do Urucum) (localidade-tipo).

45. Susuacanga octoguttata (Germar, 1821). Distribuição: Peru, Brasil (Rondônia, Maranhão, Mato Grosso, Mato Grosso do Sul, Goiás, Paraíba ao Rio Grande do Sul), Bolívia, Paraguai, Argentina (Salta, Misiones). Mato Grosso do Sul: Miranda (Salobra).

46. Uncieburia nigricans (Gounelle, 1909). Distribuição: Brasil (Piauí, Ceará, Mato Grosso, Goiás, Maranhão, Mato Grosso do Sul, Minas Gerais, São Paulo), Bolívia.

Mato Grosso do Sul: Miranda (Salobra).

\section{ECTENESSINI}

47. Ectenessa argodi Belon, 1902. Distribuição: Brasil (Mato Grosso, Mato Grosso do Sul, Goiás, Minas Gerais, São Paulo). Mato Grosso do Sul: Corumbá (Serra do Urucum); Miranda (Salobra Estrada de Ferro Noroeste do Brasil).

48. Ectenessa guttigera (Lucas, 1857). Distribuição: Brasil (Goiás, Mato Grosso do Sul, Bahia, Minas Gerais, Espírito Santo). Mato Grosso do Sul: Miranda (Salobra).

49. Ectenessa ocellata (Gounelle, 1909). Distribuição: Brasil (Piauí, Maranhão, Goiás, Mato Grosso do Sul, São Paulo), Paraguai. Mato Grosso do Sul: Coxim; Porto Murtinho; Miranda (Salobra). 50. Ectenessa quadriguttata (Burmeister, 1865). Distribuição: Brasil (Mato Grosso, Mato Grosso do Sul, São Paulo, Paraná), Bolívia, Argentina (Salta, Tucumán).

Mato Grosso do Sul: Miranda (Salobra - Estrada de Ferro Noroeste do Brasil).

51. Ectenessa villardi Belon, 1902. Distribuição: Brasil (Maranhão, Goiás, Mato Grosso, Mato Grosso do Sul, Piauí, Bahia, Minas Gerais, São Paulo), Bolívia.

Mato Grosso do Sul: Brasilândia (Rio Taquarussu); Corumbá. 52. Ectenessidia nigriventris (Belon, 1902). Distribuição: Bolívia, Brasil (Maranhão, Goiás, Mato Grosso do Sul, Bahia, Minas Gerais), Paraguai. Mato Grosso do Sul: Corumbá (Serra do Urucum); Miranda (Salobra); Porto Murtinho.

53. Ectenessidia varians (Gounelle, 1909). Distribuição: Brasil (Maranhão, Piauí ao Goiás e Mato Grosso do Sul e Minas Gerais a São Paulo), Bolívia, Paraguai. Mato Grosso do Sul: Miranda (Salobra).

54. Eurymerus eburioides Audinet-Serville, 1833. Distribuição: Brasil (Pernambuco, Minas Gerais ao Rio Grande do Sul, Goiás, Mato Grosso do Sul), Bolívia, Paraguai, Uruguai, Argentina (Salta a San Juán e Buenos Aires). Mato Grosso do Sul: sem localidade específica para o estado

55. Tricheurymerus quadristigma (Gounelle, 1909). Distribuição: Brasil (Rondônia, Goiás, Maranhão, Mato Grosso do Sul, São Paulo), Guiana Francesa, Bolívia, Paraguai, Argentina (Salta, Buenos Aires). Mato Grosso do Sul: Miranda (Salobra - Estrada de Ferro Noroeste do Brasil).

ELAPHIDIINI

56. Ambonus distinctus (Newman, 1840). Distribuição: Guiana Francesa, Brasil (Amazonas, Maranhão, Goiás, Mato Grosso, Mato Grosso do Sul, Ceará ao Rio Grande do Sul), Bolívia, Paraguai, Argentina (Misiones, Tucumán a San Luis), Uruguai. Mato Grosso do Sul: Brasilândia (Mutum), (Rio Taquarussu); Miranda (Salobra); Três Lagoas (Fazenda Beija-flor) (MarTins, 2005b:274).

57. Ambonus electus (Gahan, 1903). Distribuição: Colômbia, Suriname, Brasil (Rondônia, Mato Grosso, Goiás, Mato Grosso do Sul, Paraíba a Santa Catarina), Bolívia, Paraguai, Argentina (Salta, Jujuy, Córdoba). Mato Grosso do Sul: Corumbá (Serra do Urucum); Três lagoas (Fazenda Canaã) (Martins, 2005b:277). 58. Ambonus interrogationis (Blanchard, 1846). Distribuição: Brasil (Amazonas, Maranhão, Ceará ao Rio Grande do Sul, Goiás, Mato Grosso, Mato Grosso do Sul), Bolívia, Paraguai, Argentina. Mato Grosso do Sul: Brasilândia (Rio Taquarussu); Corumbá (Serra do Urucum); Miranda (Salobra); Três Lagoas (Fazenda Beija-Flor) (MarTins, 2005b:280).

59. Appula eduardae Franceschini, 2002. Distribuição: Brasil (Mato Grosso, Mato Grosso do Sul, Goiás). Mato Grosso do Sul: Porto Murtinho (MARTins, 2005b:45).

60. Appula lateralis (White, 1853). Distribuição: Brasil (Mato Grosso, Goiás, Mato Grosso do Sul, Bahia a Santa Catarina). Mato Grosso do Sul: Bataguassú; Brasilândia (Rio Taquarussu). 61. Eurysthea hirta (Kirby, 1818). Distribuição: Brasil (Paraíba ao Rio Grande do Sul, Goiás, Mato Grosso do Sul), Paraguai, Argentina (Misiones), Uruguai. Mato Grosso do Sul: Dourados (Martins, 2005b:349). 62. Mallocera umbrosa Gounelle, 1909. Distribuição: Brasil (Maranhão, Goiás, Mato Grosso do Sul, Minas Gerais a São Paulo), Bolívia, Paraguai, Argentina (Jujuy). Mato Grosso do Sul: Miranda (Salobra) (MARTINS, 2005b:60). 
63. Pantonyssus bitinctus Gounelle, 1909. Distribuição: Brasil (Mato Grosso, Mato Grosso do Sul, Goiás, Maranhão), Bolívia. Mato Grosso do Sul: Miranda (Salobra) (MarTins, 2005b:149). 64. Periboeum acuminatum (Thomson, 1861). Distribuição: Brasil (Pará, Mato Grosso do Sul, Rio Grande do Norte, Pernambuco, Alagoas, Bahia, Minas Gerais a Santa Catarina), Paraguai. Mato Grosso do Sul: Três Lagoas (MarTins, 2005b:227)

65. Periboeum ocellatum Gounelle, 1909. Distribuição: Brasi (Mato Grosso, Goiás, Mato Grosso do Sul, Maranhão), Bolívia, Paraguai. Mato Grosso do Sul: sem localidade específica para o estado.

66. Periboeum paucispinum (Lameere, 1890). Distribuição: Brasil (Goiás, Mato Grosso do Sul, Bahia ao Paraná), Bolívia. Mato Grosso do Sul: Brasilândia (Rio Taquarussu) (Martins, 2005b:238). 67. Periboeum piliferum (Erichson, 1847). Distribuição: Peru, Guiana Francesa, Brasil (Pará, Maranhão, Mato Grosso, Mato Grosso do Sul, Goiás, Minas Gerais ao São Paulo), Bolívia. Mato Grosso do Sul: Miranda (Salobra); Porto Murtinho (MarTins, 2005b:240).

68. Periboeum terminatum (Perroud, 1855). Distribuição: Brasil (Goiás, Mato Grosso do Sul, Bahia, Espírito Santo ao Rio Grande do Sul), Bolívia, Paraguai, Argentina.

Mato Grosso do Sul: Ponta Porã (MarTins, 2005b:244)

69. Periboeum umbrosum Gounelle, 1909. Distribuição: Brasil (Mato Grosso, Mato Grosso do Sul, Goiás, Minas Gerais), Bolívia. Mato Grosso do Sul: Miranda (Salobra) (Martins, 2005b:245). 70. Piezophidion intricatum Galileo \& Martins, 1992. Distribuição: Brasil (Mato Grosso do Sul), Bolívia, Paraguai. Mato Grosso do Sul: Miranda (Salobra); Selvíria (Fazenda da UNESP) (MARTins, 2005b:91).

71. Sphaerioeme rubristerna Martins \& Napp, 1992. Distribuição: Suriname, Brasil (Piauí, Maranhão, Mato Grosso, Goiás, Mato Grosso do Sul, Minas Gerais, São Paulo, Paraná). Mato Grosso do Sul: Coxim; Rio Brilhante (Martins, 2005b:293).

72. Sphaerion inerme White, 1853. Distribuição - Brasil (Mato Grosso, Goiás, Mato Grosso do Sul, Pernambuco, Bahia ao Rio Grande do Sul), Bolívia, Paraguai, Argentina (Jujuy, Salta), Uruguai. Mato Grosso do Sul: Bodoquena; Corumbá (Serra do Urucum); Miranda (Salobra); Porto Murtinho (Martins, 2005b:110).

73. Sphaerion lentiginosum Berg, 1889. Distribuição: Brasil (Mato Grosso do Sul), Argentina (Córdoba, Corrientes), Paraguai, Uruguai. Mato Grosso do Sul: Miranda (Salobra) (Martins, 2005b:113). 74. Sphaerion sladeni Gahan, 1903. Distribuição: Brasil (Rondônia, Mato Grosso, Goiás, Maranhão, Mato Grosso do Sul, Minas Gerais), Bolívia. Mato Grosso do Sul: Corumbá (Serra do Urucum) (MARTINS, 2005b:114).

75. Stizocera armata Audinet-Serville, 1834. Distribuição: Brasil (Mato Grosso do Sul, Bahia ao Rio Grande do Sul), Bolívia, Argentina. Mato Grosso do Sul: Miranda (Salobra) (Martins, 2005b:162).

76. Stizocera consobrina Gounelle, 1909. Distribuição: Brasil (Maranhão, Goiás, Mato Grosso do Sul, Piauí, Bahia ao Rio Grande do Sul), Paraguai. Mato Grosso do Su: Corumbá (Serra do Urucum) (MARTINS, 2005b:164).

77. Stizocera elegantula (Perroud, 1855). Distribuição: Colômbia, Brasil (Goiás, Mato Grosso do Sul, Minas Gerais, Bahia ao Rio de Janeiro), Paraguai. Mato Grosso do Sul - Brasilândia (Rio Taquarussu) (MARTINs, 2005b:169).

78. Stizocera lissonota (Bates, 1870). Distribuição: Panamá?, Brasil (Pará, Rondônia, Mato Grosso, Mato Grosso do Sul, Goiás), Guiana Francesa, Bolívia. Mato Grosso do Sul: Corumbá (Serra do Urucum) (MARTINS, 2005b:176)

79. Stizocera tristis (Guérin-Méneville, 1844). Distribuição: Brasi (Rondônia, Mato Grosso, Goiás, Piaú, Mato Grosso do Sul, Minas Gerais, São Paulo), Bolívia, Paraguai, Argentina. Mato Grosso do Sul: Corumbá (Serra do Urucum); Miranda (Salobra) (MarTins, 2005b:195)

HESPEROPHANINI

80. Anoplomerus buqueti Belon, 1890. Distribuição: Brasil (Pará, Mato Grosso, Mato Grosso do Sul, Goiás, Minas Gerais, São Paulo), Paraguai, Argentina (Formosa), Uruguai?. Mato Grosso do Sul: Corumbá (Serra do Urucum); Costa Rica (Fazenda Santo Antonio); Miranda (Salobra).

81. Eusapia amazonica (White, 1855). Distribuição: Guiana Francesa, Suriname, Brasil (Amazonas, Pará, Mato Grosso, Goiás, Mato Grosso do Sul, Maranhão, Minas Gerais a Santa Catarina), Peru, Bolívia, Paraguai, Argentina (Misiones). Mato Grosso do Sul: Miranda (Salobra).

82. Phrynocris notabilis Bates, 1867. Distribuição: Costa Rica, Guiana Francesa, Brasil (Amazonas, Pará, Maranhão, Mato Grosso, Mato Grosso do Sul, Goiás). Mato Grosso do Sul: Miranda (Salobra - Estrada de Ferro Noroeste do Brasil).

HETEROPSINI

83. Allodemus tricolor (Perty, 1832). Distribuição: Brasil (Paraíba a São Paulo, Mato Grosso do Sul), Paraguai, Argentina, Uruguai. Mato Grosso do Sul: sem localidade específica para o estado.

84. Chrysoprasis aeneiventris Bates, 1870. Distribuição: Brasil (Mato Grosso, Mato Grosso do Sul, Minas Gerais ao Rio Grande do Sul), Bolívia, Paraguai, Argentina (Jujuy, Tucumán), Uruguai. Mato Grosso do Sul: Dourados.

85. Chrysoprasis auriventris auriventris Redtenbacher, 1867. Distribuição: Brasil (Mato Grosso do Sul, Espírito Santo ao Rio Grande do Sul), Paraguai, Argentina (Misiones). Mato Grosso do Sul: Brasilândia (Rio Taquarussu).

86. Chrysoprasis basalis Chevrolat, 1859. Distribuição: Brasil (Ceará ao Rio Grande do Sul, Goiás, Mato Grosso), Bolívia, Paraguai, Argentina. Mato Grosso do Sul: Brasilândia (Rio Taquarussu); Porto Murtinho.

87. Chrysoprasis chalybea Redtenbacher, 1867. Distribuição: Brasil (Mato Grosso, Mato Grosso do Sul, Goiás, Minas Gerais ao Paraná). Mato Grosso do Sul: Porto Murtinho; Três Lagoas. 88. Chrysoprasis collaris Chevrolat, 1859. Distribuição: Brasil (Espírito Santo, Mato Grosso do Sul), Paraguai. Mato Grosso do Sul: Dourados.

89. Chrysoprasis concolor Redtenbacher, 1867. Distribuição: Brasil (Ceará, Mato Grosso, Mato Grosso do Sul, Bahia ao Rio Grande do Sul), Bolívia, Paraguai, Argentina (Misiones, Catamarca, Salta, Tucumán,Chaco, Santiago del Estero, Corrientes), Uruguai. Mato Grosso do Sul: Brasilândia (Rio Taquarussu); Dourados. 90. Chrysoprasis nigrina Bates, 1870. Distribuição: Brasil (Mato Grosso do Sul, Bahia ao São Paulo). Mato Grosso do Sul: Dourados. 91. Chrysoprasis sapphirina Gounelle, 1911. Distribuição: Brasil (Goiás, Mato Grosso do Sul, Minas Gerais), Bolívia. Mato Grosso do Sul: Rio Paraná.

92. Chrysoprasis valida Bates, 1870. Distribuição: Brasil (Mato Grosso do Sul, Minas Gerais a Santa Catarina), Bolívia. Mato Grosso do Sul: Dourados.

HEXOPLONINI

93. Hexoplon praetermissum Bates, 1870. Distribuição: Colômbia, Brasil (Pará, Goiás, Mato Grosso, Mato Grosso do Sul, Pernambuco ao Espírito Santo). Mato Grosso do Sul: Brasilândia (Rio Taquarussu) (MarTins, 2006:109).

94. Stenygra conspicua (Perty, 1832). Distribuição: Brasil (Amazonas, Pará, Mato Grosso, Mato Grosso do Sul, Paraíba ao Rio Grande do Sul), Paraguai, Argentina (Misiones, Corrientes). Mato Grosso do Sul: Corumbá (Fazenda Santa Blanca) (MARTINs,2006:36)

95. Tetroplon caudatum caudatum Aurivillius, 1899. Distribuição: Peru (?), Brasil (Amazonas (?), Mato Grosso, Goiás, Mato Grosso do Sul, São Paulo a Santa Catarina), Paraguai, Argentina (Formosa, Misiones, Chaco, Corrientes). Mato Grosso do Sul: Corumbá, Corumbá (Serra do Urucum); Sidrolândia (Vacaria); Três Lagoas (Fazenda Beija-flor). (Rio Paraná).

METHIINI

96. Methia fischeri Melzer, 1923. Distribuição: Brasil (Mato Grosso do Sul, Espírito Santo ao Rio Grande do Sul), Paraguai, Argentina (Tucumán). Mato Grosso do Sul: Corumbá (Serra do Urucum). Miranda (Salobra). NEOCORINI

97. Neocorus ibidionoides (Audinet-Serville, 1834). Distribuição: Guiana Francesa, Brasil (Goiás, Mato Grosso do Sul, Bahia ao Rio Grande do Sul), Bolívia, Paraguai, Argentina (Misiones). 
Mato Grosso do Sul: Miranda (Salobra); Três Lagoas (Fazenda Beija-flor) (MARTINs,2005a:246).

NEOIBIDIONINI

\section{NEOIBIDIONINA}

98. Coleroidion leucotrichum (Martins, 1960). Distribuição: Brasil (Mato Grosso do Sul), Argentina (Formosa, Chaco). Mato Grosso do Sul: Bodoquena; Corumbá; Miranda (Salobra) (localidade-tipo) (Martins \& Galileo, 2007:213)

99. Compsibidion campestre (Gounelle, 1909). Distribuição: Brasil (Goiás, Mato Grosso, Mato Grosso do Sul, Maranhão, Paraíba a Minas Gerais e São Paulo), Bolívia, Argentina (Jujuy, Salta, Tucumán, Santiago del Estero). Mato Grosso do Sul: Bodoquena; Corumbá (Serra do Urucum); Miranda (Salobra - Estrada de Ferro Noroeste do Brasil).

100. Compsibidion crassipede Martins, 1971. Distribuição: Brasil (São Paulo, Mato Grosso do Sul), Paraguai, Argentina (Salta). Mato Grosso do Sul: Miranda (Salobra - Estrada de Ferro Noroeste do Brasil) (localidade-tipo).

101. Ophtalmibidion luscum Martins, 1971. Distribuição: Brasil (Mato Grosso do Sul, Espírito Santo). Mato Grosso do Sul: Corumbá (localidade-tipo).

\section{COMPSINA}

102. Hadroibidion pullum (Martins, 1962). Distribuição: Brasil (Mato Grosso do Sul).

Mato Grosso do Sul: Campo Grande. Material examinado: BRASIL, Mato Grosso do Sul: Campo Grande, ổ(holótipo), 9.X.1952, M. Alvarenga col. (MNRJ).

103. Heterachthes flavicornis (Thomson, 1865). Distribuição: Brasil (Goiás, Mato Grosso do Sul, Bahia ao Rio Grande do Sul), Paraguai, Argentina (Misiones, Entre Ríos, Buenos Aires), Uruguai. Mato Grosso do Sul: Bataguassu (Rio Caraguatá $-21^{\circ} 48^{\prime} \mathrm{S}$, $\left.52^{\circ} 27^{\prime} \mathrm{W}, 400 \mathrm{~m}\right)$.

104. Kolonibidion femoratum (Martins, 1971). Distribuição: Brasil (Maranhão, Mato Grosso do Sul), Bolívia, Argentina (Chaco). Mato Grosso do Sul: Miranda (Salobra).

105. Neocompsa serrana (Martins, 1962). Distribuição: Brasil (Alagoas, Mato Grosso do Sul), Argentina (Chaco). Mato Grosso do Sul: Corumbá (Serra do Urucum).

TROPIDINA

106. Diasporidion argentinense (Martins, 1962). Distribuição: Brasil (Mato Grosso do Sul), Bolívia, Argentina (Jujuy). Mato Grosso do Sul: Bodoquena.

107. Diasporidion duplicatum (Gounelle, 1909). Distribuição: México (Morelos, Veracruz, Oaxaca, Chiapas) ao Panamá, Colômbia, Venezuela, Guiana Francesa, Brasil (Maranhão, Piauí, Goiás, Mato Grosso, Mato Grosso do Sul, São Paulo), Paraguai. Mato Grosso do Sul: Brasilândia (Rio Taquarussu); Miranda (Salobra - Estrada de Ferro Noroeste do Brasil).

108. Gnomibidion translucidum (Martins, 1960). Distribuição: Guiana Francesa, Brasil (Mato Grosso do Sul), Bolívia. Mato Grosso do Sul: Miranda (Salobra - Estrada de Ferro Noroeste do Brasil) (localidade-tipo).

109. Perissomerus hilairei hilairei Gounelle, 1909. Distribuição: Peru, Brasil (Goiás, Mato Grosso, Mato Grosso do Sul), Bolívia. Mato Grosso do Sul: Corumbá (Serra do Urucum).

110. Perissomerus hilairei bimaculatus Gounelle, 1909. Distribuição: Brasil (Mato Grosso do Sul, Bahia ao Paraná). Mato Grosso do Sul: Bataguassu (Rio Caraguatá $-21^{\circ} 48^{\prime} \mathrm{S}, 52^{\circ} 27^{\prime} \mathrm{W}$, $400 \mathrm{~m})$.

111. Thoracibidion flavopictum (Perty, 1832). Distribuição: Brasil (Goiás, Maranhão, Mato Grosso, Mato Grosso do Sul, Rio Grande do Norte a Santa Catarina), Bolívia, Paraguai, Argentina (Salta). Mato Grosso do Sul: Brasilândia (Rio Taquarussu); Corumbá; Coxim; Miranda (Salobra - Estrada de Ferro Noroeste do Brasil); Três Lagoas (Fazenda Beija-Flor).

112. Tropidion castaneum Martins, 1968. Distribuição: Brasil (Bahia, Minas Gerais, Mato Grosso do Sul), Bolívia, Paraguai. Mato Grosso do Sul: Miranda (Salobra - Estrada de Ferro Noroeste do Brasil) (localidade-tipo).

113. Tropidion flavipenne (Martins, 1964). Distribuição - Brasil (Mato Grosso, Mato Grosso do Sul). Mato Grosso do Sul: Miranda (Salobra) (localidade-tipo).
114. Tropidion inerme (Martins, 1962). Distribuição: Brasil (Mato Grosso do Sul, Rio de Janeiro ao Paraná). Mato Grosso do Sul Bataguassu (Rio Caraguatá - $21^{\circ} 48^{\prime} \mathrm{S}, 52^{\circ} 27^{\prime} \mathrm{W}, 400 \mathrm{~m}$ ).

115. Tropidion signatum punctatum Martins, 1968. Distribuição: Brasil (Goiás, Mato Grosso, Mato Grosso do Sul, Maranhão), Bolívia, Paraguai. Mato Grosso do Sul: Aquidauana; Corumbá (localidade-tipo), (Serra do Urucum); Três Lagoas (Fazenda Yamaguti)

116. Tropidion zonapterum (Martins, 1962). Distribuição: Brasil (Mato Grosso, Mato Grosso do Sul, São Paulo), Bolívia. Mato Grosso do Sul: Corumbá (Serra do Urucum); Miranda (Salobra - Estrada de Ferro Noroeste do Brasil); Três Lagoas (Fazenda Yamaguti - Córrego da Onça), (Fazenda Dr. José Mendes).

OBRIINI

117. Obrium cicatricosum Gounelle, 1909. Distribuição: Brasil (Pernambuco, Mato Grosso, Goiás, Mato Grosso do Sul, São Paulo, Rio Grande do Sul), Bolívia, Paraguai, Argentina (Salta, Chaco, Tucumán). Mato Grosso do Sul: Miranda (Salobra); Três Lagoas (Fazenda Beija-Flor).

118. Obrium multifarium Berg, 1889. Distribuição: Brasil (Mato Grosso do Sul, Minas Gerais, Rio de Janeiro, São Paulo), Paraguai, Argentina (Salta, Catamarca, Misiones). Mato Grosso do Sul: Porto Murtinho; Três Lagoas (Fazenda Yamaguti - Córrego da Onça). 119. Obrium vicinum Gounelle, 1909. Distribuição: Brasil (Pernambuco, Mato Grosso, Mato Grosso do Sul, Goiás, Bahia), Bolívia, Paraguai, Argentina (Salta). Mato Grosso do Sul - Miranda (Salobra); Porto Murtinho.

OEMINI

OEMINA

120. Aponoeme castanea Martins \& Galileo, 1997. Distribuição: Brasil (Mato Grosso do Sul). Mato Grosso do Sul: Três Lagoas (Horto Barra do Moeda, Três Lagoas Agroflorestal).

121. Argentinoeme schulzi Bruch, 1911. Distribuição: Brasil (Mato Grosso do Sul), Bolívia, Paraguai, Argentina (Córdoba). Mato Grosso do Sul: Miranda (Salobra - Estrada de Ferro Noroeste do Brasil)

122. Macroeme priapica (Thomson, 1857). Distribuição: Brasil (Pará, Goiás, Maranhão, Mato Grosso do Sul, Bahia ao Rio Grande do Sul), Bolívia, Paraguai, Uruguai, Argentina (Salta, Tucumán a Buenos Aires). Mato Grosso do Sul: Coxim; Miranda (Salobra); Três Lagoas.

123. Macroeme sobrina (Gounelle, 1909). Distribuição: Brasil (Maranhão, Goiás, Mato Grosso, Mato Grosso do Sul). Mato Grosso do Sul: Coxim.

124. Neoeme bouvieri Gounelle, 1909. Distribuição: Brasil (Pará, Goiás, Mato Grosso, Mato Grosso do Sul, Minas Gerais, São Paulo, Paraná), Guiana Francesa, Peru, Bolívia.

Mato Grosso do Sul: Bataguassu (Rio Caraguatá - $21^{\circ} 48^{\prime} \mathrm{S}$, $\left.52^{\circ} 27^{\prime} \mathrm{W}, 400 \mathrm{~m}\right)$.

125. Sphagoeme aurivillii Gounelle, 1909. Distribuição: Brasil (Maranhão, Paraíba, Mato Grosso do Sul, Minas Gerais ao Paraná), Paraguai. Mato Grosso do Sul: Miranda (Salobra).

126. Temnopis castanea Martins, 1978. Distribuição: Brasil (Mato Grosso, Mato Grosso do Sul, Bahia, Minas Gerais), Bolívia, Argentina (Salta). Mato Grosso do Sul: Miranda (Salobra).

\section{METHIOIDINA}

127. Methioeme brevipennis Zajciw, 1963. Distribuição: Brasil (Amazonas, Pará, Mato Grosso, Mato Grosso do Sul, São Paulo). Mato Grosso do Sul: Camapuã.

PIEZOCERINI

PIEZOCERINA

128. Gorybia pilosa Martins, 1976. Distribuição: Brasil (Mato Grosso do Sul)

Mato Grosso do Sul: Miranda (Salobra).

129. Gorybia ruficauda (Gounelle, 1909). Distribuição: Brasil (Maranhão, Goiás, Mato Grosso, Mato Grosso do Sul, Minas Gerais, São Paulo). Mato Grosso do Sul: Bataguassu (Rio Caraguatá

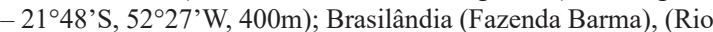
Taquarussu); Camapuã; Miranda (Salobra); Porto Murtinho. 130. Gorybia suturella Martins, 1976. Distribuição: Brasil (Mato Grosso, Mato Grosso do Sul, Maranhão), Guiana Francesa, Bolívia. Mato Grosso do Sul: Coxim. 
131. Piezarina smaragdina Martins, 1976. Distribuição: Costa Rica, Panamá, Brasil (Amazonas, Pará, Rondônia, Maranhão, Mato Grosso, Mato Grosso do Sul, Distrito Federal), Guiana Francesa. Mato Grosso do Sul: Coxim.

132. Piezocera costula Martins, 1976. Distribuição: Brasil (Mato Grosso do Sul). Mato Grosso do Sul: Corumbá; Três Lagoas (Horto Barra do Moeda, Três Lagoas Agroflorestal).

133. Thyellocerus fulgidipennis (Gounelle, 1909). Distribuição: Brasil (Amazonas, Rondônia, Goiás, Mato Grosso, Mato Grosso do Sul, São Paulo), Bolívia, Paraguai, Argentina (Salta, Misiones). Mato Grosso do Sul: Miranda (Salobra).

PTEROPLATINI

134. Deltosoma xerophila Di Iorio, 1995. Distribuição: Brasil (São Paulo, Paraná, Mato Grosso do Sul, Maranhão), Bolívia, Paraguai, Argentina (Salta, Santiago del Estero, Chaco, Córdoba). Mato Grosso do Sul: Três Lagoas (Horto Barra do Moeda) (MarTins \& NAPP, 2006).

RHINOTRAGINI

135. Eclipta nigriventris (Melzer, 1934). Distribuição: Brasil (Mato Grosso do Sul, Santa Catarina). Mato Grosso do Sul: sem localidade específica para o estado.

136. Epimelitta triangularis Fuchs, 1961. Distribuição: Brasil (Mato Grosso do Sul, Santa Catarina, Rio Grande do Sul). Mato Grosso do Sul: Bataguassu (Rio Caraguatá) (localidade-tipo). 137. Ischasioides crassitarsis (Gounelle, 1911). Distribuição: Brasil (Mato Grosso, Goiás, Mato Grosso do Sul, Bahia ao Rio Grande do Sul). Mato Grosso do Sul: Bataguassu (Rio Caraguatá). 138. Odontocera globicollis Zajciw, 1971. Distribuição: Brasil (Mato Grosso do Sul)

Mato Grosso do Sul: Nhambiquara. Material examinado: BRASIL, Mato Grosso do Sul: Nhambiquara, +(holótipo), IX-1960, M. Alvarenga col. (MNRJ).

139. Tomopterus larroides White, 1855. Distribuição: Trinidad e Tobago, Brasil (Pará, Mato Grosso, Goiás, Minas Gerais ao Rio Grande do Sul), Bolívia, Paraguai, Argentina (Salta, Tucumán, Santa Fé, Corrientes, Entre Ríos), Uruguai. Mato Grosso do Sul: sem localidade específica para o estado.

140. Tomopterus quadratipennis Bates, 1873. Distribuição: Brasil (Mato Grosso do Sul, Bahia a Santa Catarina). Mato Grosso do Sul: sem localidade específica para o estado.

RHOPALOPHORINI

141. Cosmisoma chalybeipenne Zajciw, 1962. Distribuição: Brasil (São Paulo, Mato Grosso do Sul). Mato Grosso do Sul: Bataguassu (Rio Caraguatá).

142. Cosmisoma cyaneum rubriventre Monné \& Magno, 1988. Distribuição: Brasil (Rondônia, Mato Grosso, Mato Grosso do Sul), Paraguai, Argentina. Mato Grosso do Sul: Campo Grande (Guaicurus); Maracaju.

143. Dirocoremia simplicipes (Gounelle, 1911). Distribuição: Brasil (Paraíba, Bahia, Minas Gerais, Mato Grosso do Sul, Espírito Santo, São Paulo), Bolívia, Paraguai, Argentina (Salta). Mato Grosso do Sul: Bodoquena.

144. Haenkea atra (Chevrolat, 1855). Distribuição: Brasil (Bahia Espírito Santo, Rio de Janeiro, Paraná, Santa Catarina, Mato Grosso do Sul). Mato Grosso do Sul: Dourados.

145. Ischionodonta smaragdina (Martins \& Napp, 1989). Distribuição: Brasil (Espírito Santo, Mato Grosso do Sul). Mato Grosso do Sul: Dourados.

146. Parozodes pilosus Fuchs, 1956. Distribuição: Brasil (Mato Grosso do Sul). Mato Grosso do Sul: Bataguassu (Rio Caraguatá) (localidade-tipo).

TILLOMORPHIN

147. Epropetes metallica Martins, 1975. Distribuição: Brasil (Mato Grosso do Sul). Mato Grosso do Sul: Bataguassu (Rio Caraguatá $\left.-21^{\circ} 48^{\prime} \mathrm{S}, 52^{\circ} 27^{\prime} \mathrm{W}, 400 \mathrm{~m}\right)$ (localidade-tipo).

TORNEUTINI

148. Praxithea borgmeieri Lane, 1938. Distribuição: Guiana Francesa, Brasil (Goiás, Mato Grosso, Mato Grosso do Sul, Minas Gerais). Mato Grosso do Sul: sem localidade específica para o estado.

149. Praxithea travassosi Lane, 1939. Distribuição: Costa Rica,
Venezuela, Equador, Guiana, Guiana Francesa, Brasil (Amazonas, Pará, Mato Grosso, Mato Grosso do Sul), Bolívia. Mato Grosso do Sul: Miranda (Salobra) (localidade-tipo)

150. Psygmatocerus wagleri Perty, 1828. Distribuição: Brasil (Maranhão, Alagoas ao Rio Grande do Sul), Bolívia, Paraguai, Argentina (Misiones, Tucumán, Chaco, Corrientes). Mato Grosso do Sul: Miranda (Salobra).

TRACHYDERINI

\section{TRACHYDERINA}

151. Andraegoidus rufipes (Fabricius, 1787). Distribuição: Brasil (Goiás, Mato Grosso do Sul, Paraíba ao Rio Grande do Sul), Bolívia, Paraguai, Argentina. Mato Grosso do Sul: sem localidade específica para o estado.

152. Andraegoidus fabricii Dupont, 1838. Distribuição: Brasil (Mato Grosso, Mato Grosso do Sul, Goiás, Maranhão ao Espírito Santo e são Paulo), Bolívia. Mato Grosso do Sul: Bodoquena; Miranda (Botero \& Monné, 2012).

153. Andraegoidus variegatus (Perty, 1832). Distribuição: Brasil (Pará. Mato Grosso, Goiás, Mato Grosso do Sul, Paraíba, Alagoas, Ceará ao Rio Grande do Sul), Bolívia, Paraguai, Argentina, Uruguai Mato Grosso do Sul: Bodoquena; Miranda (Botero \& MonnÉ, 2012).

154. Batus hirticornis (Gyllenhal, 1817). Distribuição: Brasil (Goiás, Mato Grosso do Sul, Bahia ao Rio Grande do Sul), Paraguai, Argentina. Mato Grosso do Sul: sem localidade específica para o estado.

155. Martinsellus signatus (Gyllenhal, 1817). Distribuição: Brasil (Goiás, Mato Grosso do Sul, Bahia ao Rio Grande do Sul), Bolívia, Paraguai, Argentina, Uruguai. Mato Grosso do Sul: sem localidade específica para o estado.

156. Metopocoilus corumbaensis Lane, 1956. Distribuição: Brasil (Pará, Tocantins, Mato Grosso, Paraíba, Ceará, Bahia, Mato Grosso do Sul). Mato Grosso do Sul: Corumbá (localidade-tipo)

157. Neochrysoprasis zajciwi Franz, 1969. Distribuição: Bolívia, Brasil (Mato Grosso, Mato Grosso do Sul, São Paulo), Paraguai. Mato Grosso do Sul: sem localidade específica para o estado.

158. Oxymerus aculeatus aculeatus Dupont, 1838. Distribuição: Brasil (Goiás, Mato Grosso, Mato Grosso do Sul, Bahia ao São Paulo, Paraná). Mato Grosso do Sul: Aquidauana (Quintino et al., 2010:365)

159. Oxymerus aculeatus meridionalis Huedepohl, 1979. Distribuição: Brasil (Mato Grosso do Sul), Bolívia, Paraguai, Argentina (Tucumán, Santiago del Estero, Santa Fé, Formosa, Chaco, Misiones, Corrientes, Entre Ríos, Buenos Aires), Uruguai. Mato Grosso do Sul: Miranda (Salobra).

160. Oxymerus bruchi bruchi Gounelle, 1913. Distribuição: Brasil (Mato Grosso, Mato Grosso do Sul, Rio Grande do Sul), Paraguai, Argentina (Tucumán, Santiago del Estero, Misiones, Chaco). Mato Grosso do Sul: Sem localidade específica para o estado.

161. Oxymerus luteus luteus (Voet, 1778). Distribuição: Brasil (Mato Grosso do Sul, Espírito Santo ao Rio Grande do Sul), Paraguai, Argentina (Santa Fé, Misiones, Chaco). Mato Grosso do Sul: Bataguassu (Rio Caraguatá).

162. Retrachydes thoracicus thoracicus (Olivier, 1790). Distribuição: Brasil (Mato Grosso, Mato Grosso do Sul, Goiás, Maranhão ao Rio Grande do Sul), Bolívia, Paraguai, Argentina, Uruguai. Mato Grosso do Sul: Sem localidade específica para o estado.

163. Retrachydes thoracicus sulcatus (Burmeister, 1865). Distribuição: Brasil (Mato Grosso do Sul), Bolívia, Paraguai, Argentina, Uruguai. Mato Grosso do Sul: Corumbá (Guiacurus); Miranda.

164. Trachelissa maculicollis (Audinet-Serville, 1834). Distribuição: Brasil (Goiás, Mato Grosso do Sul, Espírito Santo ao Rio Grande do Sul), Bolívia, Paraguai, Argentina, Uruguai. Mato Grosso do Sul: sem localidade específica para o estado. 165. Trachyderes (Trachyderes) succinctus melzeri Schwarzer, 1929. Distribuição: Brasil (Mato Grosso do Sul), Paraguai, Argentina (Salta, Chaco, Corrientes). Mato Grosso do Sul: Corumbá; Miranda, Miranda (Salobra). 
UNXIINI

166. Parunxia scopifera (Klug, 1825). Distribuição: Brasil (Espírito Santo ao Rio Grande do Sul, Mato Grosso do Sul), Uruguai. Mato Grosso do Sul: Dourados (NAPP, 2007b:315).

LAMIINAE

\section{ACANTHOCININI}

167. Lepturgantes prolatus Monné \& Monné, 2008. Distribuição: Brasil (Mato Grosso, Mato Grosso do Sul, Bahia, Espírito Santo, Minas Gerais, Rio de Janeiro, Santa Catarina), Bolívia. Mato Grosso do Sul: Corumbá (Serra do Urucum) (Monné \& MonNÉ, 2008). 168. Paratenthras martinsi Monné, 1998. Distribuição: Brasil (Mato Grosso, Mato Grosso do Sul), Bolívia. Mato Grosso do Sul: Miranda (Salobra).

ACANTHODERINI

169. Alphus similis Martins, 1985. Distribuição: Brasil (Bahia ao Rio Grande do Sul, Mato Grosso do Sul), Bolívia, Paraguai, Argentina (Formosa). Mato Grosso do Sul: Bataguassu.

170. Nesozineus bucki (Breuning, 1954). Distribuição: México, Guatemala ao Panamá, Brasil (Mato Grosso do Sul, Alagoas, Sergipe, Minas Gerais, Espírito Santo ao Rio Grande do Sul), Bolívia, Paraguai. Mato Grosso do Sul: sem localidade específica para o estado.

171. Nesozineus lineolatus Galileo \& Martins, 1996. Distribuição: Brasil (Mato Grosso do Sul, Maranhão, Alagoas, Sergipe), Paraguai. Mato Grosso do Sul: Três Lagoas (Porto Rio Verde) (localidadetipo).

172. Nesozineus triviale Galileo \& Martins, 1996. Distribuição: Brasil (Goiás, Mato Grosso do Sul, Maranhão, Bahia a Santa Catarina), Bolívia. Mato Grosso do Sul: Brasilândia (Rio Taquarussu)

173. Oreodera occulta Monné \& Fragoso, 1988. Distribuição: Brasil (Mato Grosso do Sul), Bolívia, Argentina (Formosa). Mato Grosso do Sul: Miranda. Material examinado: BRASIL, Mato Grosso do Sul: Miranda, ô(holótipo), X.1942, H. Zellibor col. (MNRJ).

174. Psapharochrus plaumanni (Fuchs, 1958). Distribuição: Brasil (Mato Grosso do Sul, Santa Catarina), Bolívia. Mato Grosso do Sul: Bataguassu (Rio Caraguatá) (Monné \& MonNÉ, 2006). 175. Steirastoma genisspina Schwarzer, 1923. Distribuição: Equador, Guiana Francesa, Brasil (Pará, Mato Grosso, Mato Grosso do Sul). Mato Grosso do Sul: Corumbá.

AERENICINI

176. Aerenica canescens (Klug, 1825). Distribuição: Brasil (Goiás, Maranhão, Mato Grosso do Sul, Tocantins, Minas Gerais, São Paulo, Paraná), Bolívia, Paraguai, Argentina (Salta, Jujuy, Chaco, Misiones). Mato Grosso do Sul: Miranda (Salobra); Porto Murtinho. 177. Aerenicopsis perforata Lane, 1939. Distribuição: Brasil (Mato Grosso do Sul, Maranhão, Paraíba a Santa Catarina), Guiana Francesa, Bolívia, Argentina (Misiones). Mato Grosso do Sul: Miranda (Salobra) (localidade-tipo).

178. Aerenomera boliviensis Gilmour, 1962. Distribuição: Bolívia, Brasil (Pernambuco, Paraíba, Bahia, Mato Grosso do Sul, Minas Gerais, São Paulo.), Paraguai. Mato Grosso do Sul: Miranda (Salobra); Ponta Porã; Porto Murtinho.

179. Antodilanea modesta (Lane, 1939). Distribuição: Brasil (Minas Gerais, Mato Grosso do Sul), Paraguai. Mato Grosso do Sul: Miranda (Salobra) (localidade-tipo).

180. Eponina flava Lane, 1939. Distribuição: Brasil (Mato Grosso do Sul, Espírito Santo, Paraná). Mato Grosso do Sul: Miranda (Salobra) (localidade-tipo).

181. Holoaerenica punctata (Gilmour, 1962). Distribuição: Brasil (Mato Grosso do Sul, São Paulo), Bolívia, Argentina (Jujuy, Salta, Catamarca, Tucumán). Mato Grosso do Sul: Miranda (Salobra). 182. Hoplistonychus bondari Melzer, 1930. Distribuição: Guiana Francesa, Peru, Brasil (Pará, Mato Grosso, Mato Grosso do Sul, Minas Gerais, São Paulo), Argentina (Misiones). Mato Grosso do Sul: Miranda (Salobra). 183. Phaula atyroa Galileo \& Martins, 2007. Distribuição: Brasil (Mato Grosso do Sul). Mato Grosso do Sul: Selvíria (localidadetipo).

184. Phaula bullula Martins, 1984. Distribuição: Brasil (Mato
Grosso do Sul). Mato Grosso do Sul: Maracaju (localidade-tipo). 185. Phaula microsticta (Lane, 1973). Distribuição: Brasil (Mato Grosso, Mato Grosso do Sul, Minas Gerais, São Paulo, Paraná), Argentina (Formosa). Mato Grosso do Sul: Costa Rica (Fazenda Santo Antônio).

186. Phaula thomsoni Lacordaire, 1872. Distribuição: Brasil (Pará ao Mato Grosso do Sul, Maranhão, Bahia, Minas Gerais ao Rio Grande do Sul), Paraguai, Argentina (Misiones). Mato Grosso do Sul: Corumbá (Fazenda Santa Blanca).

187. Pseudomecas femoralis Aurivillius, 1920. Distribuição: Brasil (Mato Grosso, Mato Grosso do Sul, Minas Gerais, Espírito Santo), Paraguai, Argentina (Santiago del Estero, Córdoba). Mato Grosso do Sul: Miranda (Salobra); Três Lagoas.

188. Recchia acutipennis (Gahan, 1889). Distribuição: Brasil (Goiás, Mato Grosso, Mato Grosso do Sul, São Paulo ao Rio Grande do Sul), Bolívia, Paraguai, Argentina (Misiones). Mato Grosso do Sul: Porto Murtinho (Várzea Alegre).

189. Recchia albicans (Guérin-Méneville, 1831). Distribuição: Brasil (Goiás, Mato Grosso do Sul, Minas Gerais a Santa Catarina), Bolívia, Argentina (Misiones). Mato Grosso do Sul: Brasilândia (Rio Taquarussu).

190. Recchia distincta (Lane, 1939). Distribuição: Brasil (Mato Grosso do Sul), Bolívia, Paraguai, Argentina (Jujuy, Santiago del Estero, Salta, Chaco, Santa Fé). Mato Grosso do Sul: Miranda (Salobra) (localidade-tipo).

191. Recchia flaveola Martins \& Galileo, 1985. Distribuição: Brasil (Goiás, Mato Grosso do Sul, Minas Gerais, São Paulo), Paraguai. Mato Grosso do Sul: Miranda (Salobra); Porto Murtinho.

192. Recchia gemignanii (Lane, 1939). Distribuição: Brasil (Goiás, Mato Grosso do Sul, Paraná), Bolívia, Paraguai, Argentina (Chaco, Tucumán). Mato Grosso do Sul: Miranda (Salobra).

193. Recchia goiana Martins \& Galileo, 1985. Distribuição: Brasil (Goiás, Mato Grosso, Mato Grosso do Sul, São Paulo), Bolívia, Paraguai. Mato Grosso do Sul: Aquidauana.

194. Recchia hirticornis (Klug, 1825). Distribuição: Venezuela, Brasil (Goiás, Mato Grosso, Mato Grosso do Sul, Pernambuco ao Rio Grande do Sul), Bolívia, Paraguai, Argentina (Salta, Jujuy, Formosa, Misiones, Chaco, Entre Ríos, Buenos Aires), Uruguai. Mato Grosso do Sul: Brasilândia (Rio Taquarussu); Campo Grande; Miranda (Salobra).

195. Recchia moema Martins \& Galileo, 1998. Distribuição: Brasil (Mato Grosso do Sul, Rio Grande do Sul), Argentina (Chaco, Córdoba, Corrientes, Entre Ríos), Uruguai. Mato Grosso do Sul: Miranda (Salobra) (localidade-tipo).

196. Recchia parvula (Lane, 1938). Distribuição: Brasil (Goiás, Mato Grosso do Sul, Minas Gerais, Rio de Janeiro, São Paulo), Bolívia. Mato Grosso do Sul: Aquidauana.

197. Recchia piriana Martins \& Galileo, 1998. Distribuição: Brasil (Mato Grosso do Sul), Paraguai. Mato Grosso do Sul: Porto Murtinho (localidade-tipo)

198. Recchia procera Martins \& Galileo, 1985. Distribuição: Brasil (Goiás, Mato Grosso do Sul, Minas Gerais ao Rio Grande do Sul), Bolívia, Paraguai, Argentina (Misiones). Mato Grosso do Sul: Bataguassu (Rio Caraguatá).

AGAPANTHIINI

199. Hippopsis (Hippopsis) prona Bates, 1866. Distribuição: Equador, Peru, Brasil (Amazônia, Goiás, Maranhão, Alagoas, Mato Grosso do Sul, Minas Gerais ao Paraná), Bolívia, Paraguai, Argentina (Chaco). Mato Grosso do Sul: sem localidade específica para o estado.

200. Trichohippopsis magna Martins \& Carvalho, 1983. Distribuição: Brasil (Goiás, Mato Grosso do Sul, Minas Gerais). Mato Grosso do Sul: Miranda (Salobra) (localidade-tipo).

APOMECYNINI

201. Catuaba sanguinolenta Martins \& Galileo, 2003. Distribuição: Brasil (Mato Grosso do Sul). Mato Grosso do Sul: Sidrolândia (Vacaria) (localidade-tipo). 202. Ptericoptus corumbaensis Galileo \& Martins, 2003. Distribuição: Brasil (Mato Grosso do Sul). Mato Grosso do Sul: Corumbá (localidade-tipo).

203. Ptericoptus dorsalis Audinet-Serville, 1835. Distribuição: 
Brasil (Pará, Paraíba, Minas Gerais, São Paulo, Mato Grosso, Mato Grosso do Sul), Bolívia. Mato Grosso do Sul: Rio Paraná.

CALLIINI

204. Callia cyanea Melzer, 1931. Distribuição: Brasil (Goiás, Mato Grosso do Sul, Minas Gerais). Mato Grosso do Sul: Sidrolândia (Vacaria).

205. Callisema rufipes Martins \& Galileo, 1990. Distribuição: Brasil (Rondônia, Maranhão, Mato Grosso, Mato Grosso do Sul, Goiás, Minas Gerais, Espírito Santo, São Paulo), Bolívia. Mato Grosso do Sul: Miranda (Salobra); Três Lagoas.

\section{COMPSOSOMATINI}

206. Aerenea quadriplagiata (Boheman, 1859). Distribuição: Brasil (Goiás, Mato Grosso do Sul, São Paulo ao Rio Grande do Sul), Bolívia, Paraguai, Argentina, Uruguai. Mato Grosso do Sul: Campo Grande; Miranda, Miranda (Salobra); Porto Murtinho.

\section{DESMIPHORIN}

207. Acaua exotica Martins \& Galileo, 1995. Distribuição: Brasil (Pará, Mato Grosso do Sul), Peru, Bolívia. Mato Grosso do Sul: Miranda (Salobra) (localidade-tipo).

208. Cicatrisestola flavicans Breuning, 1947. Distribuição: Brasil (Mato Grosso do Sul, Santa Catarina), Bolívia, Paraguai, Argentina (Chaco, Formosa). Mato Grosso do Sul: sem localidade específica para o estado.

209. Cicuiara striata (Bates, 1866). Distribuição: Venezuela, Brasil (Pará, Mato Grosso do Sul, Goiás, Minas Gerais), Bolívia. Mato Grosso do Sul: Corumbá (Serra do Urucum).

210. Cotycicuiara multifasciata Galileo \& Martins, 2008 Distribuição: Brasil (Mato Grosso, Mato Grosso do Sul, Minas Gerais). Mato Grosso do Sul: Corumbá (Serra do Urucum) (localidade-tipo) (Galileo \& Martins,2008:3)

211. Euestola basidensepunctata Breuning, 1943. Distribuição: Brasil (Maranhão, Goiás, Mato Grosso do Sul). Mato Grosso do Sul: Três Lagoas (Fazenda Retiro de Telhas).

212. Stereomerus lineatus (Breuning, 1940). Distribuição: Brasil (Goiás, Mato Grosso do Sul, Bahia, São Paulo), Bolívia. Mato Grosso do Sul: Três Lagoas (Fazenda Beija-Flor).

HEMILOPHIN

213. Adesmus hemispilus (Germar, 1821). Distribuição: Brasil (Mato Grosso do Sul, Minas Gerais ao Rio Grande do Sul), Paraguai, Argentina (Misiones). Mato Grosso do Sul: Corumbá 214. Adesmus sexlineatus (Bates, 1881). Distribuição: Brasil (Mato Grosso do Sul, Espírito Santo ao Rio Grande do Sul), Argentina (Misiones). Mato Grosso do Sul: Corumbá.

215. Canarana marceloi Martins \& Galileo, 1992. Distribuição: Brasil (Mato Grosso do Sul), Bolívia, Paraguai. Mato Grosso do Sul: Corumbá (Serra do Urucum) (localidade-tipo).

ONCIDERINI

216. Cylicasta parallela (Melzer, 1934). Distribuição: Brasil (Mato Grosso do Sul), Bolívia, Argentina (Tucumán). Mato Grosso do Sul: Campo Grande (Imbirussu); Corumbá.

217. Hesycha clavata Martins \& Galileo, 1990. Distribuição: Brasil (Mato Grosso do Sul). Mato Grosso do Sul: Dourados (localidade-tipo).

PHACELLINI

218. Neobrachychilus consobrinus (Lane, 1939). Distribuição: Brasil (Mato Grosso do Sul), Bolívia, Argentina (Salta). Mato Grosso do Sul: Miranda (Salobra) (localidade-tipo).

219. Piola quiabentiae Marinoni, 1974. Distribuição: Brasi (Maranhão, Mato Grosso do Sul, São Paulo), Bolívia, Paraguai, Argentina (Salta, Formosa). Mato Grosso do Sul: Miranda (Salobra).

POLYRHAPHIDINI

220. Polyrhaphis grandini Buquet, 1853. Distribuição: Brasil (Goiás, Mato Grosso do Sul, Minas Gerais to Santa Catarina). Mato Grosso do Sul: Ponta Porã (SANTos-Silva et al., 2010:491). 221. Polyrhaphis spinipennis Laporte, 1840. Distribuição: Brasil (Goiás. Mato Grosso, Mato Grosso do Sul, Bahia ao Rio Grande do Sul), Paraguai, Argentina, Uruguai. Mato Grosso do Sul: Bataiporã
(Fazenda Primavera); Miranda (Salobra) (SAnTos-SiLva et al., 2010:474)

TAPEININI

222. Tapeina melzeri Zajciw, 1966. Distribuição: Brasil (Mato Grosso do Sul, São Paulo, Paraná), Bolívia, Paraguai, Argentina. Mato Grosso do Sul: Bodoquena; Miranda (Salobra).

\section{XENOFREINI}

223. Xenofrea anoreina Tavakilian \& Néouze, 2006. Distribuição: Venezuela, Brasil (Pará, Goiás, Maranhão, São Paulo, Mato Grosso do Sul), Argentina (Misiones). Mato Grosso do Sul: Porto Murtinho (Fazenda Várzea Alegre) (TAVAKILIAN \& Néouze, 2006:281)

LEPTURINAE

\section{LEPTURINI}

224. Strangalia melanophthisis (Berg, 1889). Distribuição: Brasil (Mato Grosso do Sul, São Paulo ao Rio Grande do Sul), Paraguai, Argentina (Misiones, Entre Ríos), Uruguai. Mato Grosso do Sul: Campo Grande.

PARANDRINAE

PARANDRINI

225. Parandra (Parandra) glabra (DeGeer, 1774). Distribuição: México à Argentina, Antilhas (Dominica, Guadalupe, Santa Lúcia, São Vicente). Na América do Sul, Trinidad e Tobago, Venezuela, Equador, Colômbia, Peru, Bolívia, Brasil (Amazonas, Pará, Goiás, Mato Grosso, Mato Grosso do Sul, Minas Gerais, Espírito Santo, Rio de Janeiro, São Paulo ao Rio Grande do Sul), Paraguai, Argentina (Misiones). Mato Grosso do Sul: Cassilândia; Costa Rica (Fazenda Santo Antonio) (SANTOS-Silva \& Galileo, 2011:22).

PRIONINAE

ANACOLINI

226. Prionapterus travassosi Lane, 1938. Distribuição: Brasil (Mato Grosso do Sul). Mato Grosso do Sul: Maracajú (localidade-tipo).

CALOCOMINI

227. Calocomus morosus White, 1850. Distribuição: Brasil (Goiás, Mato Grosso do Sul, Minas Gerais, São Paulo), Bolívia, Paraguai, Argentina (Jujuy a Rio Negro). Mato Grosso do Sul: Terenos (Jaraguá).

228. Calocomus rugosipennis Lucas, 1857. Distribuição: Brasil (Goiás, Mato Grosso do Sul, Minas Gerais, São Paul), Argentina (Salta, Jujuy). Mato Grosso do Sul: Porto Murtinho; Terenos (Jaraguá) (SANTOS-Silva et al., 2010:69).

MALLASPINI

229. Pyrodes nitidus (Fabricius, 1787). Distribuição: Brasil (Alagoas ao Rio Grande do Sul, Mato Grosso do Sul), Paraguai, Argentina (Misiones). Mato Grosso do Sul: Bataguassu (Rio Caraguatá).

\section{DISTENIIDAE}

DISTENIINI

1. Cometes hirticornis Lepeletier \& Audinet-Serville, 1828. Distribuição: Brasil (Mato Grosso do Sul, Bahia a Santa Catarina), Paraguai, Argentina (Misiones). Mato Grosso do Sul: Bataguassu; Batayporã (Porto Primavera -Rio Paraná) (SAntos-Silva \& MARTINS, 2011:117)

2. Distenia (Basisvallis) striaticollis Villiers, 1959. Distribuição: Brasil (Mato Grosso do Sul). Mato Grosso do Sul: sem localidade específica para o estado (SANTOS-SiLVA \& MARTINS, 2011:252).

\section{VESPERIDAE}

ANOPLODERMATINAE

ANOPLODERMATINI

1. Anoploderma breueri Lameere, 1912. Distribuição: Bolívia, Brasil (Mato Grosso do Sul), Paraguai, Argentina. Mato Grosso do Sul: sem localidade específica para o estado.

2. Migdolus spitzi Lane, 1937. Distribuição: Brasil (Mato Grosso do Sul). Mato Grosso do Sul: Coxim; Porto Murtinho; Três Lagoas (Fazenda Canaã, margem esquerda do Rio Sucuriú).

MYSTERIINI

3. Pseudopathocerus humboldti (Lameere, 1912). Distribuição: Brasil (Mato Grosso do Sul), Paraguai, Argentina (Misiones). Mato Grosso do Sul: Campo Grande; Porto Murtinho (localidade-tipo), Porto Murtinho (Várzea Alegre). 
Agradecimentos. À Fundação de Apoio ao Desenvolvimento do Ensino, Ciências e Tecnologia do Estado de Mato Grosso do Sul (Fundect) e a Superintendência de Ciências e Tecnologia do Estado de Mato Grosso do Sul (Sucitec/MS) pelo convite de participação neste fascículo especial da Iheringia, Série Zoologia e o suporte financeiro para sua publicação.

\section{REFERÊNCIAS BIBLIOGRÁFICAS}

Botero, J. P. \& MonNé, M. L. 2012. Revision of the genus Andraegoidus Aurivillius (Insecta, Coleoptera, Cerambycidae). Zootaxa 3169:1-60.

Galileo, M. H. M. \& Martins, U. R. 2008. Novo gênero de Desmiphorini com cinco espécies novas (Coleoptera, Cerambycidae, Lamiinae). Les Cahiers Magellanes 83:1-10.

Lingafelter, S. W. 1998. The genera of Elaphidiini Thomson, 1864 (Coleoptera: Cerambycidae). Memoirs of the Entomological Society of Washington 20:1-118.

Martins, U. R. 2005a. Tribo Neocorini. In: Martins, U. R. org. Cerambycidae Sul-americanos (Coleoptera): Taxonomia. São Paulo, Sociedade Brasileira de Entomologia. Vol. 5, p. 239-270.

Martins, U. R. 2005b. Tribo Elaphidionini. In: Martins, U. R. org. Cerambycidae Sul-americanos (Coleoptera): Taxonomia. Curitiba, Sociedade Brasileira de Entomologia. Vol. 7., p. 1-393.

Martins, U. R. 2006. Tribo Hexoplonini. In: Martins, U. R. org. Cerambycidae Sul-americanos (Coleoptera): Taxonomia. São Paulo, Sociedade Brasileira de Entomologia. Vol. 8., p. 21-211.

Martins, U. R. \& Galileo, M. H. M. 2007. Tribo Ibidionini, Subtribo Ibidionina. In: Martins, U. R. org. Cerambycidae Sul-americanos (Coleoptera): Taxonomia. São Paulo, Sociedade Brasileira de Entomologia. Vol. 9., p. 177-330.

Martins, U. R. \& Galileo, M. H. M. 2011. Subfamília Cerambycinae, Clytini Mulsant, 1839. In: Martins, U. R. org. Cerambycidae Sulamericanos (Coleoptera). Taxonomia. São Paulo, Sociedade Brasileira de Entomologia. Vol. 12. 264p.

Martins, U. R. \& Monné, M. A. 2005. Tribo Cerambycini, Subtribo Sphallotrichina. In: Martins, U. R. org. Cerambycidae Sulamericanos (Coleoptera). Taxonomia. São Paulo, Sociedade Brasileira de Entomologia. Vol. 5., p. 1-218.

Martins, U. R. \& NAPP, D. S. 2006. Notas sobre os gêneros Deltosoma e Thelgetra (Coleoptera, Cerambycidae, Pteroplatini). Iheringia, Série Zoologia 96(3):339-344

Monné, M. A. 2006. Catalogue of the Cerambycidae (Coleoptera) of the Neotropical Region. Part III. Subfamilies Parandrinae, Prioninae, Anoplodermatinae, Aseminae, Spondylidinae, Lepturinae, Oxypeltinae, and addenda to the Cerambycinae and Lamiinae. Zootaxa 1212:1-244.

Monné, M. A. \& Monné, M. L. 2008. Synopsis of the Neotropical genus Lepturgantes Gilmour (Coleoptera: Cerambycidae) with description of a new species. Zootaxa 1876:60-68.
MonnÉ, M. L. 2005. Revisão, análise cladística e biogeografia de Coccoderus Buquet (Coleoptera, Cerambycidae). Revista Brasileira de Entomologia 49(3):369-391.

MonNÉ, M. L. \& MonNÉ, M. A. 2006. Notes on Acanthoderini (Coleoptera, Cerambycidae, Lamiinae) described by Ernst Fuchs, now in the Herbert Schmid Collection, Vienna, Austria. Zootaxa 1365:37-47.

MonNÉ, M. L. \& NAPP, D. S. 2005. Cladistic analysis of the tribe Torneutini Thomson (Coleoptera, Cerambycidae, Cerambycinae, Trachyderoinia). Zootaxa 1062:1-56.

NAPP, D. S. 1994. Phylogenetic relationships among the subfamilies of Cerambycidae (Coleoptera, Chrysomeloidea). Revista Brasileira de Entomologia 38(2):265-419.

NAPP, D. S. 2007a. Revisão do gênero Aglaoschema Napp (Coleoptera, Cerambycidae). Revista Brasileira de Zoologia 24(3):793-816.

NAPP, D. S. 2007b. Unxiini, uma nova tribo de Cerambycinae (Coleoptera, Cerambycidae). Revista Brasileira de Entomologia 51(3):312-340.

NAPP, D. S. \& Martins, U. R. 2006. Revisão do gênero Cosmoplatidius Gounelle (Coleoptera, Cerambycidae). Revista Brasileira de Zoologia 23(2):471-479.

NaPP, D. S. \& Martins, U. R. 2009. Tribo Callichromatini. In: Martins, U. R. org. Cerambycidae Sul-americanos (Coleoptera): Taxonomia. Curitiba, Sociedade Brasileira de Entomologia. Vol. 10, p. 223-352.

Philips, T. K. \& Ivie, M. A. 1998. The Methiini of the West Indies (Coleoptera: Cerambycidae), with notes on the circum-Caribbean species. Entomologica Scandinavica 29:57-87.

Quintino, H. Y.; Botero, J. P. \& MonNÉ, M. L. 2010. Insecta, Coleoptera, Cerambycidae, Cerambycinae, Trachyderini: new state and country records from South America. Check List 6(3):364-367.

Santos-Silva, A. \& Galileo. M. H. M. 2011. Sobre alguns Cerambycidae (Coleoptera) das coleções do Instituto Nacional de Pesquisas da Amazônia (INPA) e da Universidade Federal do Amazonas (UFAM): Parandrinae, Prioninae e Disteniinae. Les Cahiers Magellanes 3:20-51.

Santos-Silva, A.; Mal, N. \& Drumont, A. 2010. Revalidation of Calocomus rugosipennis Lucas, 1857 (Coleoptera, Cerambycidae, Prioninae). Bulletin de l'Institut Royal des Sciences Naturelles de Belgique 80:69-74.

Santos-Silva, A. \& Martins, U. R. 2011. Subfamília Disteniinae. In: Martins, U. R. org. Cerambycidae Sul-americanos (Coleoptera). Taxonomia. São Paulo, Sociedade Brasileira de Entomologia. Vol. 11, p. 80-255.

Santos-Silva, A.; Martins, U. R. \& Tavakilian, G. L. 2010. Revisão do gênero Polyrhaphis Audinet-Serville (Coleoptera, Cerambycidae, Lamiinae). Papéis Avulsos de Zoologia 50(30):451-509.

Tavakilian, G. L. \& NÉouZe, G. L. 2006. Materiaux por une revision des Xenofreini II. Especies nouvelles de Bolivie, Brésil, Equateur et Venezuela. Coléoptères 12(19):271-290. 